\title{
COMPORTAMENTO DE FUGA DE COLÊMBOLOS EXPOSTOS A SOLOS CONTAMINADOS COM CIPERMETRINA
}

\author{
Talyta Zortéa ${ }^{1}$, Dilmar Baretta ${ }^{2}$, Julia Corá Segat ${ }^{3}$, Ana Paula Maccari ${ }^{4}$, Carolina Riviera \\ Duarte Maluche Baretta ${ }^{5}$, Aleksandro Schafer da Silva ${ }^{2}$ \\ ${ }^{1}$ Mestranda em Zootecnia, Universidade do Estado de Santa Catarina - UDESC Oeste, e-mail: talizortea@ hotmail.com \\ ${ }^{2}$ Professor do Curso de Zootecnia e do Programa de Pós-Graduação em Zootecnia - UDESC Oeste, Bolsista em \\ Produtividade Científica (CNPq), e-mails: dilmarbaretta@gmail.com; aleksandro.silva@udsc.br \\ ${ }^{3}$ Professora Colaboradora do Curso de Zootecnia - UDESC Oeste, e-mail: juliasegat@yahoo.com.br \\ ${ }^{4}$ Doutoranda em Ciência do Solo - UDESC-CAV, e-mail: anamacc@ hotmail.com \\ ${ }^{5}$ Professora do Mestrado em Ciências Ambientais - UNOCHAPECÓ, e-mail: carolmaluche@ unochapeco.edu.br
}

RESUMO: Avaliar a toxicidade da cipermetrina para organismos do solo é uma forma de entender os efeitos deste fármaco sobre organismos não-alvo e a utilização da ecotoxicologia terrestre, que possui normas padronizadas ISO para a realização dos ensaios, permite entender esses efeitos em um âmbito mais próximo de um ecossistema natural. O presente trabalho teve como objetivo determinar o efeito da aplicação do fármaco cipermetrina no comportamento de colêmbolos da espécie Folsomia candida. Para a realização dos ensaios foi utilizado um Latossolo Vermelho distrófico proveniente da região oeste de Santa Catarina e o Solo Artificial Tropical (SAT). Os ensaios foram conduzidos em delineamento inteiramente casualizado com cinco repetições. Os tratamentos consistiam em concentrações de cipermetrina de $0 ; 7,5 ; 15 ; 22,5$ e $30 \mathrm{mg} \mathrm{kg}^{-1}$. O número de indivíduos no teste de comportamento de fuga foi analisado através do "Fisher Exact Test". Tanto o solo natural como o solo artificial apresentaram fuga de colêmbolos desde a menor dose de cipermetrina testada $\left(7,5 \mathrm{mg} \mathrm{kg}^{-1}\right)$, resultados que evidenciam a toxicidade do produto utilizado. Os valores de $\mathrm{AC}_{50}$ (Avoidance concentration - concentração efetiva de $50 \%$ de fuga) para Latossolo e SAT foram de $8,58 \mathrm{mg} \mathrm{kg}^{-1}$ e $6,47 \mathrm{mg} \mathrm{kg}^{-1}$, respectivamente. Deste modo, a presença de cipermetrina afeta negativamente o comportamento de colêmbolos $F$. candida.

Palavras-chave: ecotoxicologia terrestre, Folsomia candida, Latossolo Vermelho distrófico, piretróide.

\section{BEHAVIORAL AVOIDANCE RESPONSE OF SPRINGTAILS EXPOSED TO CONTAMINATED SOIL WITH CYPERMETHRIN}

\begin{abstract}
To evaluate the toxicity of cypermethrin for soil organisms is a way of understanding the effects of this drug on non-target organisms and the use of terrestrial ecotoxicology, which has standardized ISO standards for the tests, allows us to understand these effects in a closer context of a natural ecosystem. This study aims to determine the application of the effect of cypermethrin avoidance behavior of springtails of Folsomia candida species through escape behavior tests. For the tests we used a Oxisol from the
\end{abstract}


western region of Santa Catarina and the Tropical Soil Artificial (SAT). The tests were conducted in a completely randomized design with five replicates. The treatments consisted of cypermethrin concentrations of $0 ; 7.5 ; 15 ; 22.5$ and $30 \mathrm{mg} \mathrm{kg}^{-1}$. The number of individuals in the avoidance behavior test was analyzed by the Fisher Exact Test. Both ground natural and artificial soil were springtails escape from the lowest dose tested cypermethrin $\left(7.5 \mathrm{mg} \mathrm{kg}^{-1}\right)$ results show that the toxicity of the product used. The $\mathrm{AC}_{50}$ values for Oxisol and SAT were $8.58 \mathrm{mg} \mathrm{kg}^{-1}$ and $6.47 \mathrm{mg} \mathrm{kg}^{-1}$, respectively. Thus, the presence of cypermethrin adversely affects the $F$. candida behavior.

Keywords: terrestrial ecotoxicology, Folsomia candida, Oxisol, pyrethroid.

\section{INTRODUÇÃO}

De acordo com dados da Agência Nacional de Vigilância Sanitária (ANVISA) o Brasil é um dos países que mais movimenta o mercado de agrotóxicos no mundo (ANVISA; UFPR, 2012). Dentre os produtos químicos mais utilizados, grande parte é representada por pitretróides como a cipermetrina.

A cipermetrina tem seu uso recomendado devido a elevada eficiência no controle de ectoparasitas (carrapatos, moscas, bernes) de animais de produção e pragas em lavouras (Lagarta-do-cartucho, lagarta da soja) (LIU et al., 2011). Porém o uso inadequado de agrotóxicos pode causar desequilíbrio biológico e efeitos danosos sobre o ecossistema (SANTOS et al., 2007; REINECKE; REINECKE, 2007). Esse desequilíbrio ecológico pode ser resposta aos efeitos nos organismos não-alvo, os quais não são pragas. Na literatura podem ser encontrados trabalhos que avaliaram o efeito tóxico de alguns piretróides sobre organismos pertencentes a fauna edáfica (SECHI et al., 2014; ZORTÉA et al., 2015), abelhas, peixes (VIRAN et al., 2003), entre outros, organismos.

Com o uso em larga escala destes produtos, na produção pecuária e agrícola, como a cipermetrina, deve se ter uma preocupação com os efeitos causados em organismos do solo, pois pouco se sabe sobre tais efeitos (ZORTÉA et al., 2015), uma vez que desempenham papel importante na prestação de serviços ecossistêmicos (BARETTA et al., 2011). Para esse tipo de avaliação pode se lançar mão de uma ciência bastante difundida internacionalmente que é a ecotoxicologia terrestre. Essa ferramenta busca entender o comportamento de organismos edáficos expostos a solos contaminados com substâncias de interesse (p. ex., ISO 17512-2; 2011). Diversos testes ecotoxicológicos terrestres podem ser realizados e um dos mais utilizados, que permite resposta rápida, é o teste de comportamento de fuga com 
colêmbolos da espécie Folsomia candida (HUND-RINKE; WIECHERING, 2001; BUCH, 2010).

A realização do teste de fuga com colêmbolos vem se tornando mais frequente, pois esse teste permite resposta em curto período de tempo e indica a existência de condições desfavoráveis em um ambiente. É um teste que apresenta alta sensibilidade, podendo em alguns casos ser comparado com a sensibilidade de ensaios de reprodução e avalia efeitos subletais causados pelo contaminante (NATAL DA LUZ et al., 2004). Além disso, os organismos utilizados devem apresentar alta sensibilidade para indicar os efeitos dos contaminantes no solo, tais efeitos são dependentes do tipo de organismo ou forma de contaminação (NIEMEYER et al., 2007).

O presente trabalho teve como objetivo avaliar o comportamento de fuga de colêmbolos da espécie Folsomia candida expostos a um Latossolo Vermelho distrófico e Solo Artificial Tropical contaminados com diferentes concentrações do piretróide Cipermetrina.

\section{MATERIAL E MÉTODOS}

\subsection{SOLO TESTE}

Foram utilizadas amostras da camada superficial (0-0,20 $\mathrm{m}$ de profundidade) de um Latossolo Vermelho distrófico coletado na Empresa de Pesquisa Agropecuária e Extensão Rural (EPAGRI) no município de Chapecó - SC [2705’274” S e 0,52³8’085” W]. Também foi utilizado como solo padrão nos testes ecotoxicológicos uma mistura de $70 \%$ de areia industrial (fina), $20 \%$ de argila caulinítica, e $10 \%$ de casca de coco (moída e seca), essa mistura é conhecida como Solo Artificial Tropical (SAT) (GARCIA, 2004).

Ambos os solos foram secos em estufa a $65^{\circ} \mathrm{C}$ e tamisados em peneiras de $2 \mathrm{~mm}$, para separação de fragmentos vegetais e outros resíduos. Parâmetros físicos e químicos dos solos estão apresentados na Tabela 1 . Os solos foram corrigidos para $\mathrm{pH}$ 6,0 \pm 0,5 através da adição de carbonato de cálcio $\left(\mathrm{CaCO}_{3}\right)$ (ISO 10390, 2005) e o conteúdo de água inicial foi ajustado para 65\% da máxima capacidade de retenção de água (CRA) (ISO 11274, 1998). 


\subsection{ENSAIOS ECOTOXICOLÓGICOS}

Os tratamentos consistiram em concentrações crescentes de cipermetrina: 0 (duplo controle); 7,5; $15 ; 22,5$ e $30 \mathrm{mg} \mathrm{kg} \mathrm{kg}^{-1}$ de solo, foi utilizado o produto comercial ECTIC® com pureza de $20 \%$, no entanto as doses deste trabalho foram corrigidas para o a quantidade do principio ativo. Estas doses foram definidas a partir da recomendação que é de $3 \mathrm{~g} \mathrm{~m}^{2}$ (equivalente a $15 \mathrm{mg} \mathrm{kg}^{-1}$ ), indicada pelo fabricante do produto (ZORTÉA et al., 2015).

Os ensaios ecotoxicológicos de comportamento de fuga foram conduzidos de acordo com as normas ISO 17512-2 (2011) com delineamento inteiramente casualizado, utilizando cinco repetições. Para a montagem do experimento utilizou-se recipientes plásticos circulares (altura de $11,5 \mathrm{~cm}$ e diâmetro de $3,5 \mathrm{~cm}$ ), que foram divididos ao meio com auxílio de divisória inserida verticalmente, onde cada um dos lados recebeu $30 \mathrm{~g}$ de solo com $\mathrm{pH}$ e umidade corrigidos. Um dos lados do recipiente recebeu solo sem adição de cipermetrina e no outro lado o solo estava tratado com as respectivas concentrações do piretróide. A divisória plástica foi então retirada e 20 indivíduos com idade entre 10 e 12 dias foram colocados sobre a linha de separação formada.

Os recipientes permaneceram tampados no escuro, em ambiente com tempertaura controlada de $20{ }^{\circ} \mathrm{C} \pm 2$. O teste teve a duração de $48 \mathrm{~h}$ e os indivíduos não receberam alimentação. Após $48 \mathrm{~h}$ a divisória foi inserida verticalmente no meio do recipiente dividindoo em duas seções (solo tratado e solo não tratado) e foi contabilizado o número de indivíduos em cada seção da caixa, cada porção foi tranferida para outro recepiente onde foi adicionada água e algumas gotas de tinta preta para realização da contagem do número de indivíduos em cada seção da caixa.

Para determinar se o ensaio foi valido é necessário que os "controles duplos" não apresentem diferença de distribuição significativa entre os dois lados do recipiente teste. Para os ensaios realizados neste trabalho os "controles duplos" as duas seções do recipiente foram preenchidas com solo sem adição de cipermetrina $\left(0 \mathrm{mg} \mathrm{kg}^{-1}\right)$; e a distribuição dos indivíduos deve permanecer dentro da faixa de 40 a $60 \%$ (faixa de distribuição determinada pela ISO 17512-2 (2011). 
Tabela 1. Parâmetros físicos e químicos do Latossolo Vermelho distrófico* (LVd) e do Solo Artificial Tropical (SAT).

\begin{tabular}{|c|c|c|c|c|c|c|c|c|c|c|c|c|}
\hline & $\begin{array}{c}\mathrm{MO}^{1} \\
(\%)\end{array}$ & $\mathrm{CTC}^{2}$ & $\begin{array}{c}\text { Argila } \\
(\%)\end{array}$ & $\begin{array}{l}\mathbf{P} \\
(\mathrm{mg}\end{array}$ & $\begin{array}{r}K \\
\left.m^{-3}\right)\end{array}$ & $\begin{array}{r}\text { Ca } \\
\ldots\end{array}$ & $\begin{array}{l}\text { Mg } \\
\text { nolc d }\end{array}$ & $\begin{array}{l}\mathrm{H}+\mathrm{Al} \\
-3) \ldots\end{array}$ & $\begin{array}{l}\mathrm{Cu} \\
\ldots . . .\end{array}$ & $\mathrm{Zn}$ & $\begin{array}{c}\mathrm{Fe} \\
-3) \ldots\end{array}$ & $\begin{array}{l}\mathbf{S B}^{3} \\
\ldots .\end{array}$ \\
\hline $\mathrm{LVd}$ & 3,9 & 12,3 & 55 & 3,5 & 124 & 3,2 & 1 & 7,83 & 1,4 & 0,8 & 79,7 & 36,5 \\
\hline SAT & 10 & 77 & 20 & 28 & 0,07 & 25 & 9 & 12 & 0,2 & 6 & 1,9 & 65 \\
\hline
\end{tabular}

*Segundo o Sistema Brasileiro de Classificação dos Solos (EMBRAPA, 2006)

${ }^{1} \mathrm{MO}$ - Matéria Orgânica.

${ }^{2} \mathrm{CTC}$ - Capacidade de Troca Catiônica em pH 7,0.

${ }^{3} \mathrm{SB}$ - Soma de bases.

\subsection{ANÁLISE ESTATÍSTICA}

O número de indivíduos no teste de comportamento de fuga foi analisado através do Fischer Exact Test (ZAR, 1996), que compara o comportamento observado em relação ao comportamento esperado, ou seja, a ausência de fuga. Os valores foram gráficamente expressos e percentagem de fuga, ou seja, indivíduos que evitaram o solo contaminado. Foram estimados através do software PriProbit 1.63 (SAKUMA, 1998) os valores de $\mathrm{AC}_{50}$ (Avoidance concentration).

\section{RESULTADOS}

O teste apresentou os critérios de validação determinados pela 17512-2 (2011), onde a distribuição dos indivíduos permaneceu dentro da faixa de 40 a $60 \%$ e a mortalidade dos colêmbolos foi inferior a $20 \%$, não foram encontradas diferenças significativas $(\mathrm{P}>0,05$, Fischer Exact Test), tendo sido observada distribuição homogênea dos indivíduos nas duas seções, o que comprova que não houve preferência por um dos lados do recipiente e que o ensaio foi válido.

No Latossolo Vermelho distrófico (LVd) a fuga dos colêmbolos ocorreu já na primeira dose de cipermetrina testada $\left(7,5 \mathrm{mg} \mathrm{kg}^{-1}\right)$ (Figura 1A), enquanto que no SAT ocorreu na dose de 22,5 $\mathrm{mg} \mathrm{kg}^{-1}$ (Figura $1 \mathrm{~B}$ ). O valor de $\mathrm{AC}_{50}$ calculado para cipermetrina no $\mathrm{LVd}$ foi de 8,58 mg $\mathrm{kg}^{-1}$ e o intervalo de confiança (IC) não foi possível calcular, uma vez que as concentrações testadas são altas para este tipo de contaminante, assim ocorreu fuga 
significativa já na primeira concentração. Para o solo artificial tropical (SAT) os resultados mostraram fuga significativa apenas na terceira dose (Figura 1B) e para esse solo o $\mathrm{AC}_{50}$ calculado foi de $6,47 \mathrm{mg} \mathrm{kg}^{-1}$ (IC de 5,49=14,72 $\mathrm{mg} \mathrm{kg}^{-1}$ ).

\subsection{DISCUSSÃO}

Os resultados dos testes de fuga mostraram que a cipermetrina afeta negativamente o comportamento dos colêmbolos em ambos os solos testados, induzindo a fuga para o solo controle (Figuras 1A e 1B). Tal efeito pode ser atribuído pelo fato deste composto possuir componentes neurotóxicos, que agem no bloqueio de impulsos nervosos, assim causando paralisia rápida e morte em insetos (NARAHASHI, 1996; SANTOS et al., 2007).

Em ambos os solos houve efeito de fuga dos organismos, sendo as respostas mais sensíveis para LVd do que para o SAT (Figura 1A e 1B). Em estudo avaliando a contaminação de cambissolo com cipermetrina Zortéa et al. (2015) encontraram o valor de $\mathrm{AC}_{50}$ de $29 \mathrm{mg} \mathrm{kg}^{-1}$ para fuga de colêmbolos, valor superior aos encontrados neste estudo. Outro estudo avaliando a diversidade de colêmbolos Folsomia fimetaria em solos com aplicação de alfa-cipermetrina também indicam diminuição no número de colêmbolos em solos contaminados com doses a partir de $5 \mathrm{mg} \mathrm{kg}^{-1}$ (SECHI et al., 2014). Frampton (1999) também verificou diminuição na variabilidade espacial de colêmbolos em um campo tratado com cipermetrina, atribuindo esse resultado à fuga dos organismos.

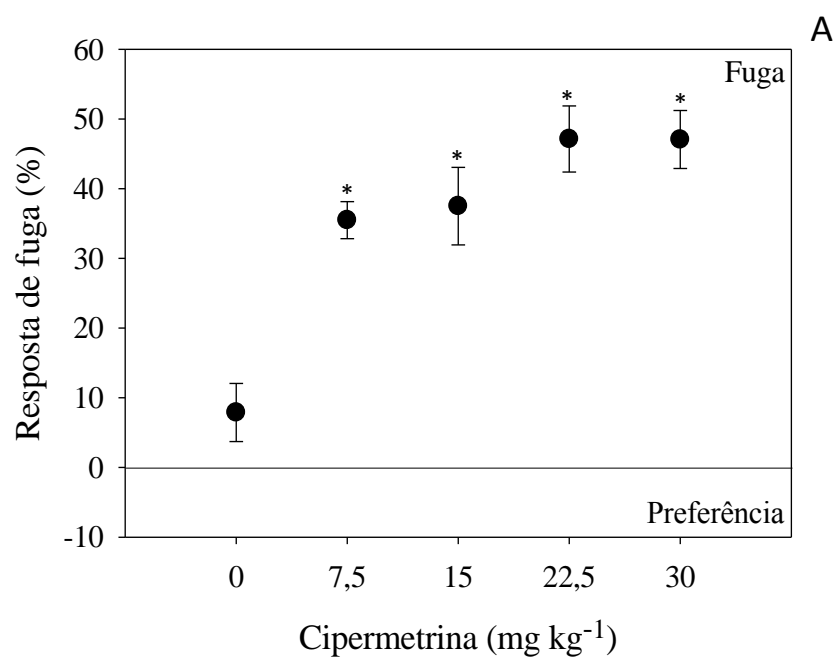




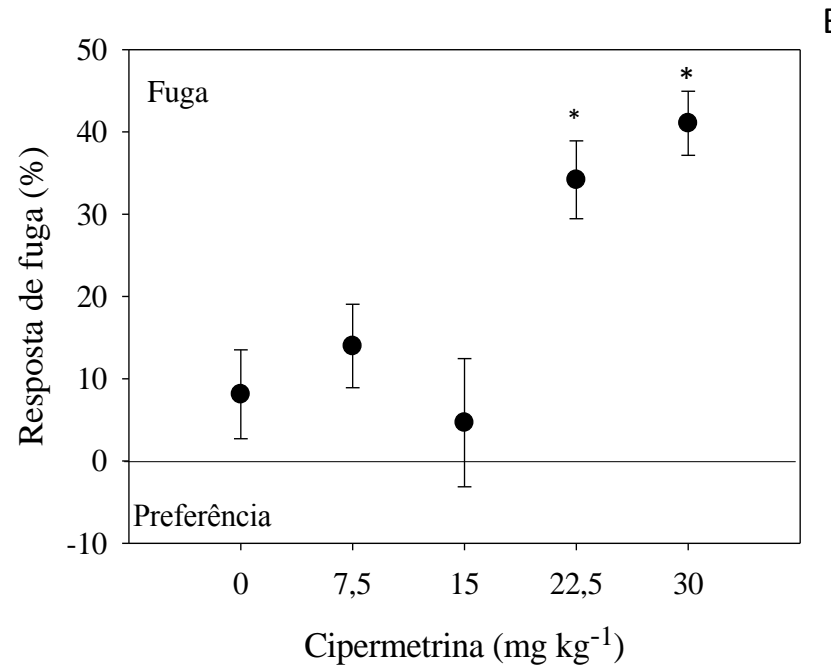

Figura 1. Percentagem de fuga (\%) de Folsomia candida no teste de comportamento de fuga em Latossolo Vermelho distrófico (A) e em solo artificial tropical (B) com crescentes doses de cipermetrina. *significativo $(\mathrm{P} \leq 0,05)$ pelo Fischer Exact Test. $(\mathrm{T})$ desvio padrão $(\mathrm{n}=5)$.

Os piretróides possuem evidente efeito tóxico para os organismos da espécie Folsomia candida, porém os valores de toxicidade são diversos entre os estudos, em especial para cipermetrina, assim é importante sempre considerar as diferenças nas características dos solos testados ou em campo, visto que, estes fatores influenciam na toxicidade dos compostos testados (ZORTÉA et al., 2015).

As diferenças nos valores de $\mathrm{AC}_{50}$ podem estar intimamente ligadas as características intrínsecas de cada tipo de solo, uma vez que, as propriedades dos solos naturais em comparação aos substratos artificiais podem alterar os resultados na avaliação de um poluente (AMORIM et al., 2005). Além disso, a diferença de toxicidade em solos naturais pode estar relacionada a fatores como, a classe textural do solo (teores de argila) e conteúdo de matéria orgânica (FILSER et al., 2014). Estes fatores atrelados atuam nos processos de adsorção dos contaminantes (metais, fármacos, pesticidas, misturas complexas), determinando os efeitos ecotoxicológicos de forma diferenciada (DOMENE et al., 2010). A utilização de solos naturais em avaliações ecotoxicológicas apresenta uma dinâmica mais realista dos possíveis efeitos causados pelo contaminante no ambiente, apontando o comportamento de organismos não alvos, que são de grande importância para o equilíbrio do ecossistema e possuem funções específicas na manutenção e qualidade do ambiente terrestre (NUNES; ESPÍNDOLA, 2012). 


\section{CONCLUSÕES}

Ensaios de curta duração (fuga) para verificar os efeitos de contaminantes do solo, pode ser uma alternativa rápida de avaliação de efeitos e um indicador potencial de condições adversas em um determinado ambiente. Assim, com base nos resultados apresentados pelos testes de fuga com colêmbolos, conclui-se que a presença de cipermetrina afeta negativamente o comportamento desta espécie, uma vez que, é capaz de e promove a fuga dos indivíduos em concentrações a partir de 7,5 $\mathrm{mg} \mathrm{kg}^{-1}$ no Latossolo Vermelho distrófico, enquanto para o solo artificial tropical a fuga pode ser observada a partir de $22,5 \mathrm{mg} \mathrm{kg}^{-1}$.

\section{REFERÊNCIAS}

AMORIM, M. J.; RÖMBKE, J.; SCHALLNA, H. J.; SOARES, A. M. V. M. Effect of soil properties and aging on the toxicity of copper for improving soil tests with earthworms, springtails, plants Enchytraeus albidus, Enchytraeus luxuriosus, and Folsomia candida. Environmental Toxicology and Chemistry, New York, v. 24, p. 1875-1885, 2005.

ANVISA; UFPR. Seminário de mercado de agrotóxico e regulação. Brasilia: ANVISA, 2012.

BARETTA, D.; SANTOS, J.C.P.; SEGAT, J.C.; GEREMIA, E.V.; OLIVEIRA FILHO, L.C.I.; ALVES, M.V. Fauna edáfica e qualidade do solo. Tópicos Ciência do Solo. 2011;8:119-70.

BUCH, A. C. Pontoscolex corethrurus (Müller, 1857) e Eisenia andrei, Bouché 1972, como bioindicadoras de solos contaminados por agrotóxicos. 2010. 61 f. Dissertação (Mestrado em Ciência do Solo) - Universidade Federal do Paraná, Curitiba, 2010.

DOMENE, X.; CHELINHO, S.; SOUSA, J. P. Role of soil properties in sewage sludge toxicity to soil collembolans. Soil Biology and Biochemistry, Oxford. v. 42, p. 1982-1990, 2010 .

FILSER, J.; WIEGMANN, S.; SCHRÖDER, B. Collembola in ecotoxicology-Any news or 
just boring routine? Applied Soil Ecology, Amsterdam, v. 83, p. 193-199, 2014.

FRAMPTON, G. K., Spatial variation in non-target effects of the insecticides chlorpyrifos, cypermethrin and pirimicarb on Collembola in winter wheat. Pesticide Science, sl. v. 55, p. 875-886, 1999.

GARCIA, M.V. Effects of pesticides on soil fauna: development of ecotoxicological test methods for tropical regions. Ecology and Development Series, Germany: University of Bonn, 2004. 281p.

HUND-RINKE, K.; WIECHERING, H. Earthworm avoidance test for soil assessment. Journal of Soils and Sediments, sl., v. 1, p. 15-20, 2001.

ISO International Organization for Standardization. ISO - 10390: Soil quality Determination of pH. Geneva, Switzerland, 2005. 7p.

ISO International Organization for Standardization. ISO - 17512-2. Soil quality - Avoidance test for determining the quality of soils and effects of chemicals on behaviour - Part 2: Test with collembolans (Folsomia candida). Genève, Switzerland, 2011. 13p.

ISO International Organization for Standardization. ISO-11274: Soil quality - Determination of the water-retention characteristic - laboratory methods. Genève, Switzerland, 1998. 36p.

LIU, S.; ZHOU, Q. X.; WANG, Y. Y. Ecotoxicological responses of the earthworm Eisenia fetida exposed to soil contaminated with HHCB. Chemosphere, Oxford, v. 83, p. 1080-1086, 2011.

NARAHASHI, T. Neuronal ion channel as the target sites of insecticides. Pharmacology and Toxicology, sl., v.79, n.1, p.1-14, 1996.

NATAL DA LUZ, T.; RIBEIRO, R.; SOUSA, J. P. Avoidance tests with collembola and earthworms as early screening tools for site specific assessment of polluted soils. Environmental Toxicology and Chemistry, New York, v.23, p. 2188-2193, 2004.

NIEMEYER, J. C.; SILVA, E. M.; SOUSA, J. S. Desenvolvimento de um Esquema para Avaliação de Risco Ecológico em Ambientes Tropicais: Estudo de Caso da Contaminação por Metais em Santo Amaro da Purificação, Bahia, Brasil. Journal of the Brazilian Society of Ecotoxicology, Itajaí. v. 2, p. 263-267, 2007. 
NUNES, M. E. T.; ESPÍNDOLA, E .L. G. Sensitivity of Eisenia andrei (Annelida, Oligochaeta) to a commercial formulation of abamectina in avoidance tests with artificial substrate and natural soil under tropical conditions. Ecotoxicology, Londres, v. 2, p. 1063-71, 2012

REINECKE, S. A.; REINECKE, A. J. The impact of organophosphate pesticides in orchards on earthworms in the Western Cape, South Africa. Ecotoxicology and Environmental Safety, Paris, v. 66, p. 244-251, 2007.

SAKUMA, M. Probit analysis of preference data. Applied Entomology and Zoology. Tokyo, v.33, n.3, p. 339-347, 1998.

SANTOS, M. A. T.; AREAS, M. A.; REYES, F. G. R. Piretróides - uma visão geral. Alimentos e Nutrição, Araraquara, v. 18, n. 3, p. 339-349, 2007.

SECHI, V.; D’ANNIBALE, A.; MARALDO, K.; JOHANSEN, A.; BOSSI, R.; JENSEN, J.; KROGH, P.H. Species composition of a soil invertebrate multi-species test system determines the level of ecotoxicity. Environmental and Pollution, sl. v. 184, p. 586-596, 2014.

VIRAN, R.; FIGEN, Ü. E.; HILAL, P.; ONER, K. Investigation of acute toxicity of deltamethrin on guppies (Poecilia reticulata). Ecotoxicology and Environmental Safety, Paris, v.55, p.82-85, 2003.

ZAR, J. Biostatistical Analysis. 3.ed. London: Prentice-Hall. 1996. 663p.

ZORTÉA, T.; BARETTA, D.; MACCARI, A. P.; SEGAT, J. C.; BOIAGO, E. S.; SOUSA, J. P.; SILVA, A. S. Influence of cypermethrin on avoidance behavior, survival and reproduction of Folsomia candida in soil. Chemosphere, Oxford, v. 122, p. 94-98, 2015. 